\title{
Astragalosides attenuate learning and memory impairment in rats following ischemia-reperfusion injury
}

\author{
YANG-YANG WU*, WANG-YANG WU**, HUI-LING GONG, WEI-ZU LI and YAN-YAN YIN \\ Department of Pharmacology, Anhui Medical University, Key Laboratory of Chinese Medicine Research and Development, \\ State Administration of Traditional Chinese Medicine, Hefei, Anhui 230032, P.R. China
}

Received June 6, 2013; Accepted February 3, 2014

DOI: $10.3892 / \mathrm{mmr} .2014 .1969$

\begin{abstract}
Astragalosides (ASTs) have been traditionally used in the treatment of various cardiovascular and cerebrovascular diseases. The aim of the present study was to investigate the neuroprotective effects of AST on learning and memory following focal cerebral ischemia/reperfusion in a rat model. A Morris water maze was used to measure the effect of AST on learning and memory impairments. A histological examination and Hoechst 33258 staining was used to observe the neuronal changes and apoptosis in the hippocampus. The activity of phospho-extracellular signal-regulated kinases (p-ERK), p-c-Jun N-terminal kinases (JNK) and p-Akt was measured by western blotting. The data revealed that AST improved the rats learning and memory abilities, attenuated neuronal cells apoptosis, increased the expression of p-ERK and p-Akt, and decreased the expression of p-JNK. These findings indicated that AST has protective effects that may be correlated with the inhibition of neuronal cell apoptosis and the regulation of p-ERK, p-Akt and p-JNK expression.
\end{abstract}

\section{Introduction}

Ischemic brain injury is a common cause of permanent disability and is associated with dementia and cognitive decline in the elderly (1). Certain surgical procedures that involve the reduction or interruption of the blood supply to the brain and events such as strokes, are often accompanied by memory loss that persists for several months during recovery. These changes are believed to be associated with focal cerebral ischemia. The middle cerebral artery occlusion model (MCAO) that was originally developed in rats

Correspondence to: Professor Yan-Yan Yin, Department of Pharmacology, Anhui Medical University, Key Laboratory of Chinese Medicine Research and Development, State Administration of Traditional Chinese Medicine, 81 Meishan Road, Hefei, Anhui 230032, P.R. China

E-mail: yinyanyan5678@126.com

*Contributed equally

Key words: astragalosides, cerebral ischemia, ischemia/reperfusion, learning and memory, mitogen-activated protein kinase is considered to be a reliable and reproducible model. The model induces deficits in cognitive function in the rats, which appear to remain fairly stable $(2,3)$.

Neurotrophic factors are known to be critical in neurite outgrowth and cell survival. Nerve growth factor (NGF) and brain-derived neutrophic factor (BDNF) may bind to acceptors, stimulate the downstream signaling pathways and protect rat hippocampal neurons against ischemic cell damage (4). Mitogen-activated protein kinase (MAPK) family members, including extracellular signal-regulated kinases (ERK)1/2, p38 MAPK and c-Jun N-terminal kinase (JNK), respond to various extracellular stimuli, thereby transmitting extracellular signals into the nucleus. The JNK signaling pathway is a potential cascade mediating neuronal apoptosis triggered by focal ischemia (5). While, the MEK/ERK pathway is important in cell growth and differentiation following ischemia, activation of the Akt kinase pathway by growth factors also has neuroprotective effects (6).

Astragalus is a traditional Chinese medicine, also known as legumes Mongolia astragalus or the dried root of Astragalus membranaceus (Fisch.) Bge. Astragalosides (ASTs) are the main component of Astragalus and function as antioxidants, in immune regulation and to promote intelligence. ASTs have been commonly used in the prevention and treatment of cardiovascular and cerebrovascular diseases, aging, immune function disorders and other diseases. Our previous studies revealed that ASTs have protective effects against ischemic damage $(7,8)$ and improve behavioral disorders, including problems with spatial learning and memory in AD rats (9); therefore, we hypothesize that AST may improve the learning and memory abilities of rats following ischemia reperfusion (I/R) injury. On the basis of the association between cognitive decline and AST, the current study was performed to observe whether AST regulates the behavioral disorder in rats following ischemia, and its possible mechanism.

\section{Materials and methods}

Reagents. AST brown powder (content, $>95 \%$ AST) was provided by the Institute of Anhui Hengxing Medicine (Hefei, China). Ginaton (Gin) was provided by Willmar Schwabe Pharmaceuticals (Karlsruhe, Germany). Hochest 33258 was provided by Sigma (St. Louis, MO, USA). ERK, phospho (p)-ERK, JNK, p-JNK, Akt and p-Akt monoclonal antibodies 
were obtained from Santa Cruz Biotechnology, Inc. (Santa Cruz, CA, USA).

Animals. Sprague-Dawley rats (weight, 250-325 g) were supplied by The Experimental Animal Center of Anhui Medical University (Hefei, China). Animals were housed four per cage, allowed access to water and food ad libitum and maintained in a constant temperature of $22 \pm 2^{\circ} \mathrm{C}$, with a humidity of $50 \pm 10 \%$ under a $12 \mathrm{~h} \mathrm{light/dark} \mathrm{cycle.} \mathrm{Animal}$ treatment and maintenance was conducted in accordance with the guidelines for the humane treatment of animals set by the association of Laboratory Animal Sciences and The Center for Laboratory Animal Sciences at Anhui Medical University.

Rat I/Rmodel and treatment. The MCAO surgery was conducted according to previously described methods (10). The rats were anesthetized by $10 \%$ chloral hydrate $(300 \mathrm{mg} / \mathrm{kg}$, intraperitonealy) and placed in dorsal recumbency. A longitudinal incision of $1.5 \mathrm{~cm}$ in length was made in the midline of the ventral cervical skin. The right common carotid artery, internal carotid artery (ICA) and external carotid artery (ECA) were exposed and carefully isolated. A nylon monofilament $(40 \mathrm{~mm}$ in length and $0.24 \mathrm{~mm}$ in diameter), its tip rounded by flame-heating, was inserted from the lumen of the ECA to that of the right ICA to occlude the origin of the right middle cerebral artery (MCA). The right MCA was occluded for $120 \mathrm{~min}$, following which, the cerebral blood flow was restored by withdrawal of the nylon thread.

As a means of assessing the adequacy of the occlusion, a neurological score was assigned to each animal 5 min prior to removing the occlusion and at $22 \mathrm{~h}$ after reperfusion: 0 , no deficit; 1 , forelimb weakness; 2 , circling to the affected side, 3 , partial paralysis on the affected side; and 4, no spontaneous motor activity. Following $120 \mathrm{~min}$ of occlusion, all the animals were randomly assigned according to the neurological function deficit score (11) to one of the following groups: I/R, AST $(20,40$ and $80 \mathrm{mg} / \mathrm{kg}$ ) or Gin $(40 \mathrm{mg} / \mathrm{kg})$. The rats were administered treatment by gavage at 0,8 and $24 \mathrm{~h}$ reperfusion, then once a day for 7 days. Sham-control animals were prepared in the same way, with the exception of the insertion of a nylon surgical thread into the right ICA. The animal body temperature was maintained at $37 \pm 1^{\circ} \mathrm{C}$ during and following the surgery. The animals were sacrificed by decapitation following a Morris water maze (MWM) test at reperfusion day 7 , and the brain was used for immunohistochemistry.

Another batch of rats was randomly divided into the following five groups: Sham, I/R, Gin (40 mg/kg) and AST (40 and $80 \mathrm{mg} / \mathrm{kg}$ ). These rats were administrated treatment by gavage once a day for 7 days. After the last intragastric administration, the rats were underwent MCAO for $120 \mathrm{~min}$, then the thread was withdrawn and the blood flow for was restored for $22 \mathrm{~h}$, before the rats were sacrificed. A neurological score was assigned to each animal 5 min prior to removing the occlusion and at $22 \mathrm{~h}$ post-reperfusion. At $22 \mathrm{~h}$ after reperfusion, the rats were sacrificed and the brains were dissected, frozen in powdered dry ice and stored at $-80^{\circ} \mathrm{C}$ until further use for western-blotting.

MWM test. An MWM was used to test spatial learning and memory, and was performed on days 4,5,6 and 7 post-ischemia.
The maze consisted of a black circular pool $2.14 \mathrm{~m}$ in diameter and $80 \mathrm{~cm}$ in height, filled with water at $21-22^{\circ} \mathrm{C}$ to a height of $50 \mathrm{~cm}$. A black circular platform that was $9 \mathrm{~cm}$ in diameter was placed $2.0 \mathrm{~cm}$ below the water line in the center of one quadrant and remained in the same position. Several, constant, large visual cues surrounded the tank at a height of $120-150 \mathrm{~cm}$ to facilitate orientation. The rat was placed in the water facing the wall at one of four random start locations (north, south, east and west, locating at equal distances from each other on the pool rim). Each rat was allowed to locate the submerged platform within $90 \mathrm{sec}$ and rest on it for $20 \mathrm{sec}$. If the rat failed to locate the hidden platform within $90 \mathrm{sec}$, it was placed on it for $20 \mathrm{sec}$. The procedure was repeated for all the four starting locations. The latency to reach the platform and the swimming speed were recorded, each representing the average of four trials. The escape latency, i.e. the time to reach the platform, and the length of the path the animal swam to find the platform were used to assess the acquisition of the water-maze task. The shorter the latency to locate the platform, the better the rats memory for its location was considered to be.

Histological examination. For the histological examination, the rats were perfused transcardially with normal saline, followed by fixation with $4 \%$ paraformaldehyde. The brains were removed and stored in the same paraformaldehyde solution. Serial paraffin sections were cut coronally on a Leica microtome (Mannheim, Germany). The sections were stained with hematoxylin and eosin (H\&E) and examined under a light microscope (Olympus, Tokyo, Japan).

Hoeschst 33258 staining. For nuclear staining, the paraffin sections were deparaffinized with xylene (Beyotime, Shanghai, China) twice for $15 \mathrm{~min}$ at $37^{\circ} \mathrm{C}$, washed with phosphate-buffered saline (PBS), mounted onto slides using antifade mounting medium (Beyotime,) and then examined by fluorescence microscopy (Olympus). Morphologically, cells undergoing apoptosis should appear smaller than normal, with chromatin that appears condensed and deeply stained. Cellular fragmentation into apoptotic bodies also occurs.

Western blotting. The rats were sacrificed by decapitation at a specified time under anesthesia. The infarcted brains were separated, frozen quickly in liquid nitrogen and stored at $-80^{\circ} \mathrm{C}$. The tissues were homogenized in ice-cold homogenization buffer (HB; Beyotime). Following the protein concentration measurement using the Lowry method, with bovine serum used as a standard, each sample was diluted to equal protein concentrations with HB. Next, 4X sodium dodecyl sulfate-polyacrylamide gel electrophoresis (SDS-PAGE) sample buffer was added into the sample, which was then boiled in a $100^{\circ} \mathrm{C}$ waterbath for $10 \mathrm{~min}$. Protein $(50 \mu \mathrm{g})$ was loaded onto each lane, separated by $15 \%$ SDS-PAGE and transferred onto a polyvinylidene difluoride membrane. The membrane was blocked with $5 \%$ skimmed milk for $2 \mathrm{~h}$ and then probed with p-ERK1/2 (1:1,000; 9101; Cell Signaling, Beverly, MA, USA) or ERK1/2 (1:1,000; 9102; Cell Signaling, Beverly, MA, USA) at $4^{\circ} \mathrm{C}$ overnight. The detection was performed using horseradish peroxidase-conjugated goat anti-rabbit $\mathrm{IgG}$ and developed using $0.05 \%$ 3,3'-diaminobenzidine in PBS containing $0.01 \%$ $\mathrm{H}_{2} \mathrm{O}_{2}$. The bands on the membrane were scanned and analyzed 
Table I. Effects of AST on the escape latency during the training session in the MWM test in I/R rats (mean \pm standard deviation, $\mathrm{n}=8)$.

\begin{tabular}{lccccc}
\hline & & \multicolumn{4}{c}{ Latency, sec } \\
\cline { 3 - 6 } Group & Dose, mg/kg & 4 days & 5 days & 6 days & 7 days \\
\hline Sham & - & $70.92 \pm 17.31$ & $60.70 \pm 15.42$ & $47.55 \pm 11.99$ & $33.57 \pm 9.86$ \\
Model & - & $94.19 \pm 18.47^{\mathrm{a}}$ & $85.29 \pm 15.02^{\mathrm{b}}$ & $79.84 \pm 18.40^{\mathrm{b}}$ & $67.81 \pm 15.60^{\mathrm{b}}$ \\
Gin & 40 & $78.42 \pm 22.21$ & $73.88 \pm 15.25$ & $60.22 \pm 16.17^{\mathrm{c}}$ & $48.90 \pm 7.71^{\mathrm{d}}$ \\
AST & 20 & $79.87 \pm 20.61$ & $70.48 \pm 19.50$ & $64.64 \pm 12.62$ & $51.85 \pm 9.04^{\mathrm{c}}$ \\
& 40 & $78.01 \pm 22.48$ & $75.63 \pm 12.80$ & $61.34 \pm 15.73^{\mathrm{c}}$ & $49.05 \pm 6.73^{\mathrm{d}}$ \\
& 80 & $75.59 \pm 20.86$ & $69.59 \pm 13.19^{\mathrm{c}}$ & $55.78 \pm 18.34^{\mathrm{c}}$ & $46.01 \pm 13.65^{\mathrm{d}}$ \\
\hline
\end{tabular}

${ }^{\text {aP }}<0.05$ and ${ }^{\mathrm{b}} \mathrm{P}<0.01$ vs. the sham group; ${ }^{\mathrm{C}} \mathrm{P}<0.05$ and ${ }^{\mathrm{d}} \mathrm{P}<0.01$ vs. the model group. ASTs, astragalosides; Gin, ginaton; I/R, ischemia/reperfusion; MWM, morris water maze.

Table II. Effects of AST on the swim distance during the training session in the MWM in I/R rats (mean \pm standard deviation, $\mathrm{n}=8)$.

\begin{tabular}{lccccc}
\hline & & \multicolumn{4}{c}{ Distance, cm } \\
\cline { 3 - 6 } Group & Dose, $\mathrm{mg} / \mathrm{kg}$ & 4 days & 5 days & 6 days & 7 days \\
\hline Sham & - & $1808.19 \pm 661.94$ & $1502.32 \pm 503.84$ & $1221.18 \pm 307.44$ & $833.62 \pm 173.04$ \\
Model & - & $2982.97 \pm 746.86^{\mathrm{a}}$ & $2648.16 \pm 473.43^{\mathrm{a}}$ & $2312.10 \pm 425.62^{\mathrm{a}}$ & $1763.64 \pm 524.13^{\mathrm{a}}$ \\
Gin & 40 & $2312.00 \pm 589.51$ & $2065.43 \pm 445.45^{\mathrm{b}}$ & $1551.98 \pm 404.00^{\mathrm{c}}$ & $1113.76 \pm 224.62^{\mathrm{c}}$ \\
AST & 20 & $2558.83 \pm 552.58$ & $2254.27 \pm 565.87$ & $1862.86 \pm 411.14^{\mathrm{b}}$ & $1217.34 \pm 290.95^{\mathrm{b}}$ \\
& 40 & $2303.95 \pm 693.06$ & $2060.13 \pm 451.50^{\mathrm{b}}$ & $1528.46 \pm 431.47^{\mathrm{c}}$ & $1083.47 \pm 260.98^{\mathrm{c}}$ \\
& 80 & $2258.92 \pm 640.11$ & $1875.59 \pm 481.58^{\mathrm{c}}$ & $1423.50 \pm 377.50^{\mathrm{c}}$ & $967.20 \pm 270.72^{\mathrm{c}}$ \\
\hline
\end{tabular}

${ }^{\mathrm{a}} \mathrm{P}<0.01$ vs. the sham group; ${ }^{\mathrm{b}} \mathrm{P}<0.05$ and ${ }^{\mathrm{c}} \mathrm{P}<0.01$ vs. the model group. AST, astragalosides; $\mathrm{I} / \mathrm{R}$, ischemia/reperfusion; MWM, morris water maze; Gin, ginaton.

by Eaglesight software (Stratagene, La Jolla, CA, USA). The proteins were visualized by an enhanced chemiluminescence system (Bioshine, Shanghai, China).

Statistical analysis. The data were expressed as the mean \pm standard deviation. Significant differences between groups were determined by a one-way analysis of variance and the t-test. $\mathrm{P}<0.05$ was used to indicate a statistically significant difference.

\section{Results}

Effects of AST on learning and memory impairment in $I / R$ rats. To measure the correlation between the brain I/R injury and cognitive deficits and the protective effects of AST in rats, learning and memory was assessed by the MWM test. At each day of observation of the MWM test, the ischemic rats spent more time locating the submerged platform when compared with the sham rats. At days 4, 5 and 6, the AST $(20 \mathrm{mg} / \mathrm{kg})$-treated ischemic rats showed a trend towards locating the platform in a shorter time relative to the ischemic group. The AST $(40 \mathrm{mg} / \mathrm{kg})$-treated group exhibited a short- ened latency at day 6 , while AST $(80 \mathrm{mg} / \mathrm{kg}$ ) also had the same effect at days 5 and 6 . At the last testing day (day 7), AST (20, $40,80 \mathrm{mg} / \mathrm{kg}$ ) markedly shortened the time it took to locate the hidden platform compared with the ischemic rats $(\mathrm{P}<0.05$ or $\mathrm{P}<0.01$ ) (Table I).

The length of the path the rats swam to locate the platform was also recorded to assess the acquisition of the water-maze task. Compared with the sham group, the $\mathrm{I} / \mathrm{R}$ group rats swam a longer distance to locate the platform $(\mathrm{P}<0.01)$. AST $(40 \mathrm{mg} / \mathrm{kg})$ shortened the distance at day 6 , and AST (40 mg/kg) had the same action at days 5 and 6. On the last testing day, AST $(20,40,80 \mathrm{mg} / \mathrm{kg})$ markedly shortened the distance swam when compared with the I/R group $(\mathrm{P}<0.01)$ (Table II).

Effect of AST on neuronal degenerative changes and apoptosis in the hippocampus of $I / R$ rats. To investigate the correlation between memory impairment and neuronal degeneration and apoptosis, the neuropathology and levels of apoptosis were measured (Fig. 1). The model group demonstrated neuron degeneration in the hippocampus (CA1), whereas no evident neuronal abnormalities were present in the control group. The 


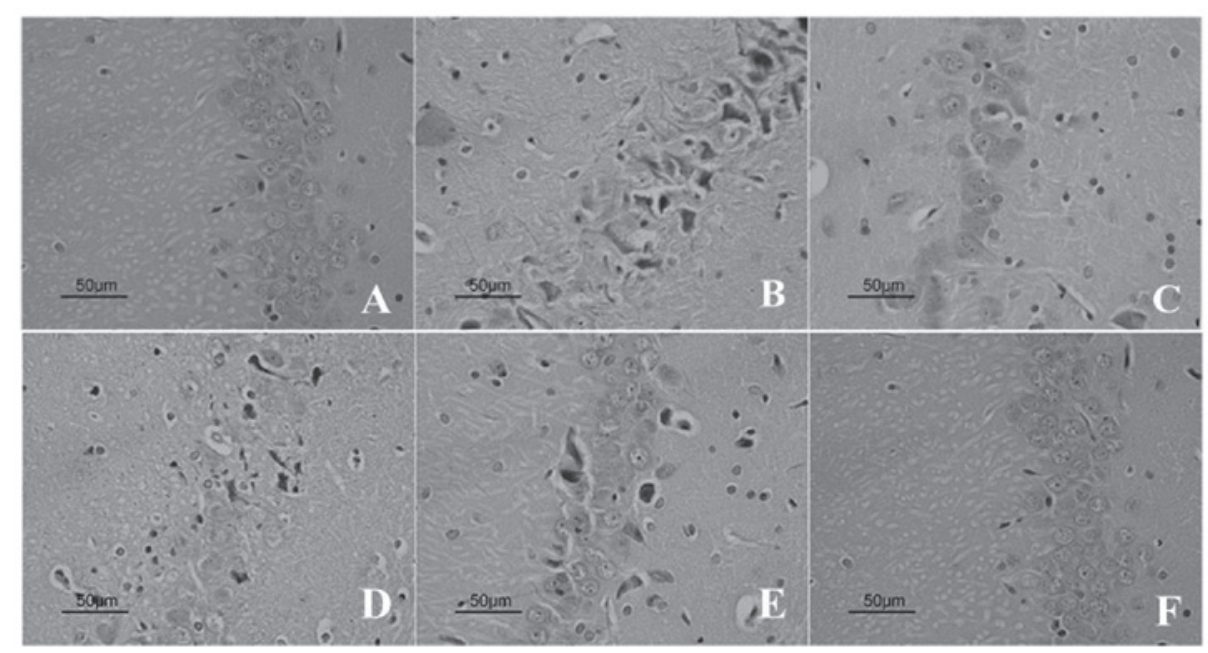

Figure 1. Histopathological observation of the hippocampus (CA1; hematoxylin and eosin stain). No neuronal abnormabilities in the hippocampus (CA1) of the sham group, were observed, whereas the I/R group rats showed degeneration of the neurons in the hippocampus (CA1) and the neurons were disarrayed. AST and Gin markedly improved the pathomorphological changes of the hippocampus (CA1). (A) Sham; (B) I/R (model); (C) Gin (40 mg/kg); (D) AST (20 mg/kg); (E) AST (40 mg/kg) and (F) AST (80 mg/kg) (original magnification, x400). AST, astragalosides; I/R, ischemia/reperfusion; Gin, ginaton.
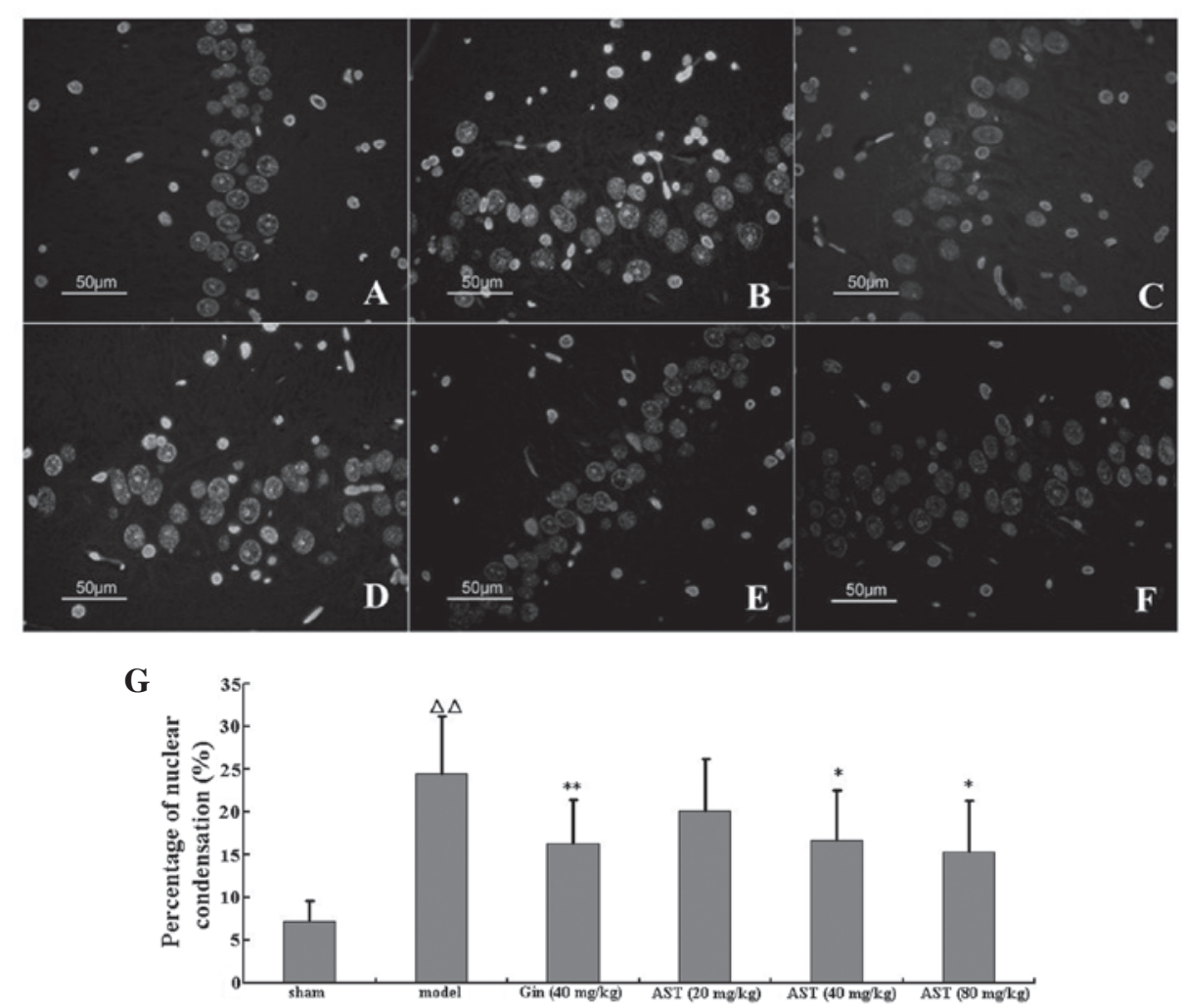

Figure 2. (A-F) Photomicrographs of hippocampal (CA1) neurons stained with Hoechst 33258 following I/R in rats. Nuclear condensation and fragmentation were prominent after I/R. (A) Sham; (B) model; (C) Gin (40 mg/kg); (D) AST (20 mg/kg); (E) AST (40 mg/kg) and (F) AST (80 mg/kg) (original magnification, $\mathrm{x} 400)$.(G) The percentage of nuclear condensed cells in the hippocampal (CA1) neurons was counted. Compared with the sham group, the I/R group exhibited a significantly increased percentage of nuclear condensed cells. AST and Gin decreased the percentage. ${ }^{\Delta \Lambda} \mathrm{P}<0.01$ vs. Sham group; ${ }^{*} \mathrm{P}<0.05$ vs. model group; ${ }^{* * *} \mathrm{P}<0.01$ vs. model group. AST; astragalosides; Gin, ginaton; I/R, ischemia/reperfusion.

neuronal cell bodies became small and deeply stained with dye. AST may improve the pathomorphological changes of the hippocampal neurons and reduce the neuronal chromatin, which is condensed.

Hippocampal neuronal apoptosis was explored further by staining with Hoechst 33258 , which binds to chromatin and allows fluorescent visualization of normal and condensed chromatin. Morphologically, the cells undergoing apoptosis became small and deeply stained and cellular fragmentation into apoptotic bodies occured (Fig. 2A). Compared with the control group, the percentage of condensed cells in the hippocampus increased in the MCAO rats, whereas AST significantly decreased the percentage in the hippocampus (CA1) (Fig. 2B). 
Table III. Effect of AST on the neurological function evaluation of rats following I/R injury (mean \pm standard deviation, $\mathrm{n}=8)$.

\begin{tabular}{lccc}
\hline & & \multicolumn{2}{c}{ Neurological deficit score } \\
\cline { 3 - 4 } Group & $\begin{array}{r}\text { Dose, } \\
\mathrm{mg} / \mathrm{kg}\end{array}$ & $2 \mathrm{~h}$ & $22 \mathrm{~h}$ \\
\hline Sham & - & $0.00 \pm 0.00$ & $0.00 \pm 0.00$ \\
Model & - & $2.38 \pm 0.52$ & $2.63 \pm 0.52$ \\
Gin & 40 & $2.25 \pm 0.71$ & $1.88 \pm 0.64^{\mathrm{a}}$ \\
AST & 40 & $2.50 \pm 0.53$ & $2.00 \pm 0.53^{\mathrm{a}}$ \\
& 80 & $2.13 \pm 0.64$ & $1.88 \pm 0.35^{\mathrm{b}}$ \\
\hline
\end{tabular}

${ }^{\mathrm{a}} \mathrm{P}<0.01$ vs. I/R group. ${ }^{\mathrm{b}} \mathrm{P}<0.01$ vs. model group. AST, astragalosides; $\mathrm{I} / \mathrm{R}$, ischemia/reperfusion; Gin, ginaton.

Effect of AST on the neurological function evaluation of $M C A O$ rats. A neurological deficit score may indicate the neurological function impairment of rats following ischemia reperfusion injury. AST $(40,80 \mathrm{mg} / \mathrm{kg})$ significantly reduced the neurological deficit score when compared with the I/R group, indicating that pretreatment with AST may improve the neurological disorder induced by cerebral I/R (Table III).

Effects of AST on the activity of $p$-ERK, $p$-JNK and $p$-Akt in the brains of $I / R$ rats. To further analyze the cell signaling pathways responsible for the AST neuroprotective effects, western blotting was performed with tissue samples obtained from the brain tissue of the ischemic rats. As shown in Fig. 3, the bands for $\mathrm{p}-\mathrm{ERK}, \mathrm{p}-\mathrm{JNK}$ and $\mathrm{p}$-Akt were observed in each group. The level of p-ERK, p-JNK and p-Akt was quantified by densitometry. There was increased phosphorylation of ERK and Akt, and decreased phosphorylation of JNK in the I/R group; pretreatment with AST (40 or $80 \mathrm{mg} / \mathrm{kg}$ ) may activate ERK and Akt phosphorylation while depressing JNK phosphorylation further.

\section{Discussion}

The development of mazes to investigate spatial learning and memory has provided a method to determine the protective effects of drugs on the behavioral consequences and the neurological damage to the hippocampus and other areas involved in the neurotoxic effects of ischemia (12). The deficits on the learning and memory of rats following I/R injury was therefore measured by MWM in the present study. MCAO induced a failure in the spatial memory function of the rats when they were tested on days 4 to 7 following the surgery. This result demonstrated that AST shortened the latency and distance of swimming compared with the I/R group, which indicated that AST was able to diminish the abnormal behavioral performance following I/R. Gin is extracted from the leaves of Ginkgo biloba and is a famous Chinese traditional medicine used for treating cerebrovascular diseases and Alzheimer's disease, therefore it was selected as a positive control. The data revealed that Gin may shorten the latency and distance of swimming, similar to the effects of AST.
A

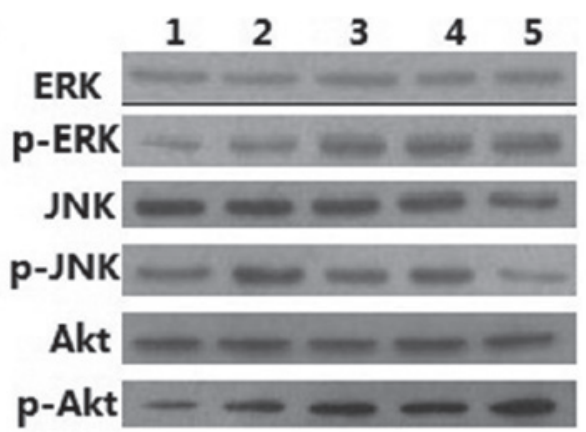

B

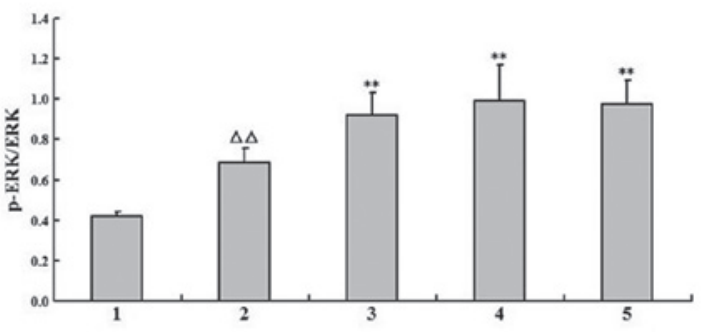

C

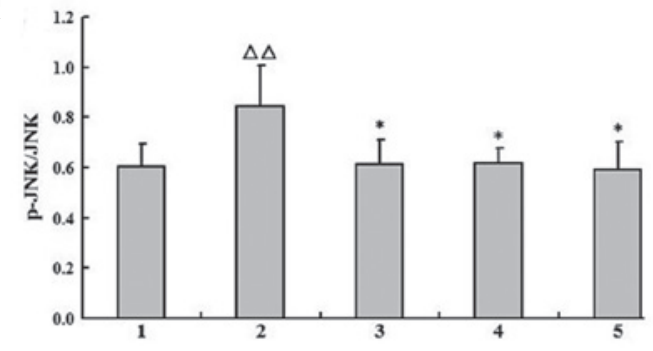

D

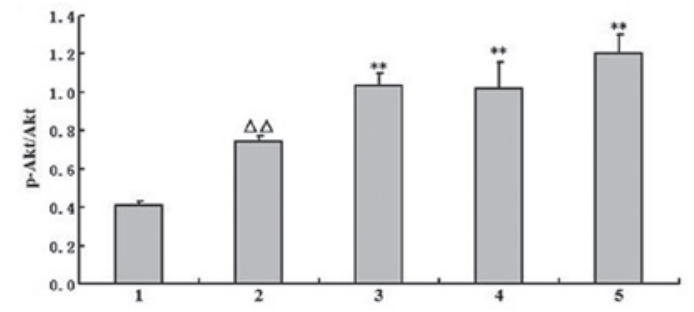

Figure 3. Effects of AST on the activity of p-ERK, p-Akt and p-JNK in the $\mathrm{I} / \mathrm{R}$ rats (mean \pm standard deviation, $\mathrm{n}=5$ ). (A) Representative western blotting of ERK, p-ERK, JNK, p-JNK, Akt and p-Akt in cerebral ischemia tissue from the different groups of rats at $22 \mathrm{~h}$ post-reperfusion. (B, C and D). The data of the (B) p-ERK/ERK, (C) p-JNK/JNK and (D) p-Akt/Akt. 1, sham; 2, model; 3, Gin (40 mg/kg); 4, AST (40 mg/kg) and 5, AST $(80 \mathrm{mg} / \mathrm{kg})$. The presented data are based on at least three independent experiments $\left({ }^{\Delta} \mathrm{P}<0.01\right.$ vs. sham group; ${ }^{*} \mathrm{P}<0.05$ and ${ }^{* *} \mathrm{P}<0.01$ vs. model group by Fisher's LSD post-hoc after one-way analysis of variance). AST, astragalosides; ERK, extracellular signal-regulated kinases; I/R, ischemia/reperfusion; Gin, ginaton; p-, phospho.

The I/R injury induced neuronal apoptosis and learning and memory impairments, therefore, the protective effects of AST on neuronal apoptosis in the hippocampus were investigated further. Apoptosis is a subtype of cell death that is involved in diverse physiological and pathological processes (13). In the present study, a histological examination revealed that the neuronal cell bodies became short and deeply stained with dye following I/R injury, and nuclear staining with Hoechst 33258 displayed nuclear condensation and fragmentation in the cells undergoing apoptosis. AST may ameliorate these pathological 
changes and inhibit the apoptosis in the hippocampus in MCAO rats. This may be the protective mechanism of AST on I/R injury.

Our previous data demonstrated that AST may increase the expression of NGF and BDNF and the mRNA expression of TrkA and TrkB, while decreasing the p75 NTR mRNA level $(7,8)$. Therefore we hypothesize that AST may produce a marked effect through growth factors and downstream signaling pathways. The ERK, JNK and Akt signaling pathways may regulate neuronal survival and apoptosis following ischemia, but the majority of studies agree that the levels of p-ERK, p-JNK and p-Akt decrease after $24 \mathrm{~h}$ or more following ischemia $(14,15)$, therefore the level of $\mathrm{p}-\mathrm{ERK}$, p-JNK and p-Akt was detected $22 \mathrm{~h}$ after reperfusion in the present study. The p-ERK levels were enhanced following use of a variety of protective agents, such as BDNF and erythropoietin (16-18). The phosphorylation of ERK is an essential component in inducing neuroprotection. The serine/threonine kinase, Akt, also known as protein kinase B, enhances survival with cerebral ischemia through a PI3-kinase-dependent signaling pathway. PI3K/Akt signaling is also important in neurogenesis. In addition, Akt is a critical mediator of cellular responses to growth factors, whereas JNK may be a key mediator for the transmission of apoptotic signals to mitochondrial apoptosis-related proteins $(19,20)$. The JNK signaling pathway has been demonstrated to be activated following focal cerebral ischemia. Furthermore, ischemia-induced JNK activity may promote neuronal apoptosis (21). In the present study, the changes in ERK, JNK and Akt phosphorylation were measured in the rats treated with AST, and the results revealed that AST was able to increase ERK and Akt phosphorylation and decrease the expression of p-JNK following I/R.

In conclusion, the present study indicated that AST may attenuate learning and memory impairments in rats following ischemia-reperfusion, and that this may be associated with the regulation of ERK, JNK and Akt phosphorylation and their expression levels.

\section{Acknowledgements}

This study was supported by grants from The Nature Science Foundation of Anhui Province Education (grant nos. KJ2008B301 and KJ2009A81), Young Teachers in Colleges and Universities Provincial Research Projects of Anhui Province (grant no. 2008jq1059zd).

\section{References}

1. Hattori K, Lee H, Hurn PD, Crain BJ, Traystman RJ and DeVries AC: Cognitive deficits after focal cerebral ischemia in mice. Stroke 31: 1939-1944, 2000.

2. Bouët V, Freret T, Toutain J, Divoux D, Boulouard M and Schumann-Bard P: Sensorimotor and cognitive deficits after transient middle cerebral artery occlusion in the mouse. Exp Neurol 203: 555-567, 2007.

3. Shioda N, Han F, Morioka $M$ and Fukunaga K: Bis(1-oxy-2-pyridinethiolato)oxovanadium(IV) enhances neurogenesis via phosphatidylinositol 3-kinase/Akt and extracellular signal regulated kinase activation in the hippocampal subgranular zone after mouse focal cerebral ischemia. Neuroscience 155: 876-887, 2008
4. Lee TH, Kato H, Chen ST, Kogure K and Itoyama Y: Expression of nerve growth factor and trkA after transient focal cerebral ischemia in rats. Stroke 29: 1687-1697, 1998.

5. Irving EA and Bamford M: Role of mitogen- and stress-activated kinases in ischemic injury. J Cereb Blood Flow Metab 22: 631-647, 2002.

6. Zhao H, Shimohata T, Wang JQ, Sun G, Schaal DW, Sapolsky RM and Steinberg GK: Akt contributes to neuroprotection by hypothermia against cerebral ischemia in rats. J Neurosci 25: 9794-9806. 2005

7. Yin YY, Li WP, Gong HL, Zhu FF, Li WZ and Wu GC: Protective effect of astragaloside on focal cerebral ischemia/reperfusion injury in rats. Am J Chin Med 38: 517-527, 2010.

8. Yin YY, Li WP, Li WZ, Gong HL, Zhu FF and Wu GC: Effects of astragalosides on the expression of BDNF, TrkB and p75NTR mRNA against focal cerebral ischemia-reperfusion injury. Chinese Pharmacological Bulletin 25: 672-676, 2009.

9. Li W, Li W, Yin Y, Gong H, Wu G and Zhu F: Protective effects of AST and ASI on memory impairment and its mechanism in senescent rats treated by GC. Zhongguo Zhong Yao Za Zhi 34: 199-203, 2009 (In Chinese).

10. Longa EZ, Weinstein PR, Carlson S and Cummins R: Reversible middle cerebral artery occlusion without craniectomy in rats. Stroke 20: 84-91, 1989.

11. Bederson JB, Pitts LH, Tsuji M, Nishimura MC, Davis RL and Bartkowski H: Rat middle cerebral artery occlusion: evaluation of the model and development of a neurologic examination. Stroke 17: 472-476, 1986

12. Wiard RP, Dickerson MC, Beek O, Norton R and Cooper BR: Neuroprotective properties of the novel antiepileptic lamotrigine in a gerbil model of global cerebral ischemia. Stroke 26: 466-472, 1995.

13. Li WZ, Li WP, Zhang W, Yin YY, Sun XX, Zhou SS, Xu XQ and Tao CR: Protective effect of extract of Astragalus on learning and memory impairments and neurons' apoptosis induced by glucocorticoids in 12-month-old male mice. Anat Rec (Hoboken) 294: 1003-1014, 2011

14. Kim SJ, Yoo KY, Jeong CW, Kim WM, Lee HK, Bae HB, Kwak SH, Li M and Lee J: Urinary trypsin inhibitors afford cardioprotective effects through activation of PI3K-Akt and ERK signal transduction and inhibition of p38 MAPK and JNK. Cardiology 114: 264-270, 2009.

15. Wang PR, Wang JS, Zhang C, Song XF, Tian N and Kong LY: Huang-Lian-Jie-Du-Decotion induced protective autophagy against the injury of cerebral ischemia/reperfusion via MAPK-mTOR signaling pathway. J Ethnopharmacol 149: 270-280, 2013.

16. Zhang F, Wang S, Signore AP and Chen J: Neuroprotective effects of leptin against ischemic injury induced by oxygen-glucose deprivation and transient cerebral ischemia. Stroke 38: 2329-2336, 2007.

17. Kilic E, Kilic U, Soliz J, Bassetti CL, Gassmann M and Hermann DM: Brain-derived erythropoietin protects from focal cerebral ischemia by dual activation of ERK-1/-2 and Akt pathways. FASEB J 19: 2026-2028, 2005.

18. Lange-Asschenfeldt C, Raval AP, Dave KR, Mochly-Rosen D, Sick TJ and Pérez-Pinzón MA: Epsilon protein kinase C mediated ischemic tolerance requires activation of the extracellular regulated kinase pathway in the organotypic hippocampal slice. J Cereb Blood Flow Metab 24: 636-645, 2004.

19. Tournier C, Hess P, Yang DD, Xu J, Turner TK, Nimnual A, Bar-Sagi D, Jones SN, Flavell RA and Davis RJ: Requirement of JNK for stress-induced activation of the cytochrome c-mediated death pathway. Science 288: 870-874, 2000.

20. Lei K, Nimnual A, Zong WX, Kennedy NJ, Flavell RA, Thompson CB, Bar-Sagi D, Davis RJ: The Bax subfamily of Bcl2-related proteins is essential for apoptotic signal transduction by c-Jun NH(2)-terminal kinase. Mol Cell Biol 22: 4929-4942, 2002.

21. Okuno S, Saito A, Hayashi T, Chan PH: The c-Jun N-terminal protein kinase signaling pathway mediates Bax activation and subsequent neuronal apoptosis through interaction with Bim after transient focal cerebral ischemia. J Neurosci 24: 7879-7887, 2004. 